\title{
Remarkable genomic diversity among Escherichia isolates recovered from healthy chickens
}

\author{
Nicholas M Thomson ${ }^{1}$, Rachel Gilroy ${ }^{1}$, Maria Getino ${ }^{2,3}$, Ebenezer Foster-Nyarko ${ }^{1,4}$, Arnoud HM van Vliet ${ }^{3}$, \\ Roberto M La Ragione ${ }^{3,5}$, Mark J Pallen ${ }^{\text {Corresp. 1, 3, } 6}$ \\ 1 Quadram Institute Bioscience, Norwich, Norfolk, United Kingdom \\ 2 NIHR Health Protection Research Unit in Healthcare Associated Infections and Antimicrobial Resistance, Department of Infectious Disease, Imperial \\ College London, London, United Kingdom \\ 3 Department of Pathology and Infectious Diseases, School of Veterinary Medicine, University of Surrey, Guildford, Surrey, United Kingdom \\ 4 Department of Infection Biology, London School of Hygiene \& Tropical Medicine, University of London, London, United Kingdom \\ 5 Department of Microbial Sciences, School of Biosciences and Medicine, University of Surrey, Guildford, Surrey, United Kingdom \\ 6 School of Biological Sciences, University of East Anglia, Norwich, Norfolk, United Kingdom \\ Corresponding Author: Mark J Pallen \\ Email address: mark.pallen@quadram.ac.uk
}

The genus Escherichia has been extensively studied and it is known to encompass a range of commensal and pathogenic bacteria that primarily inhabit the gastrointestinal tracts of warm-blooded vertebrates. However, the presence of $E$. coli as a model organism and potential pathogen has diverted attention away from commensal strains and other species in the genus. To investigate the diversity of Escherichia in healthy chickens, we collected fecal samples from antibiotic-free Lohmann Brown layer hens and determined the genome sequences of 100 isolates, 81 of which were indistinguishable at the HCO level of the Hierarchical Clustering of Core Genome Multi-Locus Sequence Typing scheme. Despite initial selection on CHROMagar Orientation medium, which is considered selective for $E$. coli, in silico phylotyping and core genome single nucleotide polymorphism analysis revealed the presence of at least one representative of all major clades of Escherichia, except for $E$. albertii, Shigella, and E. coli phylogroup B2 and cryptic clade I. The most frequent phylogenomic groups were $E$. coli phylogroups $A$ and $\mathrm{B} 1$ and $E$. ruysiae (clades III and IV). We compiled a collection of reference strains isolated from avian sources (predominantly chicken), representing every Escherichia phylogroup and species, and used it to confirm the phylogeny and diversity of our isolates. Overall, the isolates carried low numbers of the virulence and antibiotic resistance genes typically seen in avian pathogenic E. coli. Notably, the clades not recovered are ones that have been most strongly associated with virulence by other studies. 
1 Remarkable genomic diversity among Escherichia 2 isolates recovered from healthy chickens

3

4

Nicholas M. Thomson ${ }^{1}$, Rachel Gilroy ${ }^{1}$, Maria Getino ${ }^{2,3}$, Ebenezer Foster-Nyarko ${ }^{1,4}$, Arnoud H.M. van Vliet ${ }^{2}$, Roberto M. La Ragione ${ }^{2,5}$ and Mark J. Pallen ${ }^{1,2,6 \#}$

${ }^{1}$ Quadram Institute Bioscience, Norwich Research Park, Norwich, Norfolk, United Kingdom

2 Department of Pathology and Infectious Diseases, School of Veterinary Medicine, University of Surrey, Guildford, Surrey, United Kingdom ${ }^{3}$ (Current address) NIHR Health Protection Research Unit in Healthcare Associated Infections and Antimicrobial Resistance, Department of Infectious Disease, Faculty of Medicine, Imperial College London, London, United Kingdom

${ }^{4}$ (Current address) Department of Infection Biology, London School of Hygiene and Tropical Medicine, London, United Kingdom

${ }^{5}$ Department of Microbial Sciences, School of Biosciences and Medicine, University of Surrey, Guildford, Surrey, United Kingdom

${ }^{6}$ University of East Anglia, Norwich Research Park, Norwich, Norfolk, United Kingdom

${ }^{\#}$ Corresponding author: Professor Mark J. Pallen, Quadram Institute Bioscience, Norwich Research Park, Norwich, Norfolk, NR4 7UQ, United Kingdom Email: Mark.Pallen@quadram.ac.uk; m.pallen@uea.ac.uk

\section{Abstract}

The genus Escherichia has been extensively studied and it is known to encompass a range of commensal and pathogenic bacteria that primarily inhabit the gastrointestinal tracts of warmblooded vertebrates. However, the presence of $E$. coli as a model organism and potential pathogen has diverted attention away from commensal strains and other species in the genus. To investigate the diversity of Escherichia in healthy chickens, we collected fecal samples from antibiotic-free Lohmann Brown layer hens and determined the genome sequences of 100 isolates, 81 of which were indistinguishable at the $\mathrm{HC} 0$ level of the Hierarchical Clustering of Core Genome Multi-Locus Sequence Typing scheme. Despite initial selection on CHROMagar Orientation medium, which is considered selective for E. coli, in silico phylotyping and core 
genome single nucleotide polymorphism analysis revealed the presence of at least one

36

37 representative of all major clades of Escherichia, except for E. albertii, Shigella, and E. coli phylogroup B2 and cryptic clade I. The most frequent phylogenomic groups were E. coli phylogroups A and B1 and E. ruysiae (clades III and IV). We compiled a collection of reference strains isolated from avian sources (predominantly chicken), representing every Escherichia phylogroup and species, and used it to confirm the phylogeny and diversity of our isolates.

Overall, the isolates carried low numbers of the virulence and antibiotic resistance genes typically seen in avian pathogenic E. coli. Notably, the clades not recovered are ones that have been most strongly associated with virulence by other studies.

\section{Introduction}

Members of the genus Escherichia are common inhabitants of the gastrointestinal tracts of warm-blooded animals (Tenaillon et al., 2010; Jang et al., 2017). Although many isolates are believed to be beneficial or harmless components of a healthy microbiome, the genus has received a large share of research attention as some members—notably certain strains of Escherichia coli_cause disease and/or carry transferable antibiotic resistance genes (Kaper, Nataro \& Mobley, 2004; Cummins et al., 2019).

Much effort has been expended to develop techniques for identifying and categorizing members of Escherichia and understanding the population structure of the genus. Classical phenotypic techniques identified species by morphology and biochemical tests and divided strains into pathovars, based on site of infection, e.g., uropathogenic E. coli (UPEC) causing urinary tract infections; and serotypes, based on antibody recognition of variation in lipopolysaccharides, flagella and fimbriae (Fratamico et al., 2016). These methods have gradually given way to genome-based approaches classifying isolates with multi-locus enzyme electrophoresis studies

(Selander et al., 1987) and multi-locus sequence typing (Maiden et al., 1998). Whole genome 
61 sequencing technologies have recently facilitated the comparison of strains in ever greater

62 detail (Uelze et al., 2020), leading to new classification schemes based on average nucleotide

63 identity (Konstantinidis \& Tiedje, 2005) and whole-genome nucleotide polymorphism (Schürch

64 et al., 2018).

65

66 The avian pathogenic E. coli (APEC) pathovar causes colibacillosis in chickens, turkeys and

67 other avian species, and is responsible for significant morbidity and mortality in the worldwide

68 poultry industry (Dziva \& Stevens, 2008). Certain serotypes and virulence factors are frequently

69 associated with APEC but it remains challenging to distinguish categorically between

70 commensal and virulent strains (Mehat, van Vliet \& La Ragione, 2021).

71

72 The large number of high-quality Escherichia genomes from diverse geographical and biological sources has forced a re-thinking of classification. Thus, although Shimwellia blattae was initially

74 placed within Escherichia, it is now assigned to a separate genus (Priest \& Barker, 2010). By contrast, although named as if a genus, Shigella is now thought merely to represent a series of pathovars of E. coli (Yang et al., 2005; Devanga Ragupathi et al., 2018). Nevertheless, the assignment of strains of $E$. coli to seven phylogroups (A, B1, B2, C, D, E and F) has proven a robust finding that holds for phylogenetic trees built from core genome alignments of large numbers of isolates. Phylogroups $\mathrm{G}$ and $\mathrm{H}$ have recently been proposed (Lu et al., 2016; Clermont et al., 2019), while five of what were initially called 'cryptic clades' (Walk et al., 2009) have been assigned to species: Clade I to E. coli (Clermont et al., 2013), Clade II to E. whittamii

82 (Gilroy et al., 2021), Clades III and IV together to E. ruysiae (van der Putten et al., 2021), and 83 Clade V to E. marmotae (Liu et al., 2015).

84

85 The accumulation of genomic data for Escherichia has been strongly biased towards clinical 86 isolates from humans and economically important animals (Touchon et al., 2020). This hampers 
87 efforts to achieve a complete understanding of Escherichia diversity, ecology, and population

88 genetics. Here, we report phylogenomic analyses on 100 Escherichia isolates cultured from

89 fecal samples from a small flock of healthy adult layer chickens, 81 of which we determined to

90 be unique.

91

92 Materials \& Methods

93 Sample collection and storage. Fresh fecal samples from healthy adult Lohmann Brown layer

94 hens on a farm in the south-east of England were collected to isolate presumptive E. coli. The

95 birds (flock size $=24$ ) were purchased from a single flock in April 2017 at 20 weeks old and kept

96 in a large outdoor run with a substrate of stone chippings and small turf enrichment beds during

97 the day and in a coop overnight. They were fed Farmgate Layer pellets and Mash (ForFarmers

98 Ltd, Bury St Edmunds, UK), according to the manufacturer's instructions, and no antibiotics

99 were used. Sampling was carried out between July and November 2018. Once a month, five

100 freshly voided fecal samples were collected from different hens. 1:10 serial dilutions of each

101 sample were plated (100 $\mu \mathrm{L})$ onto MacConkey agar to obtain single colonies. Several colonies

102 with different morphologies were selected and streaked to fresh MacConkey agar plates to

103 isolate single colonies. Identification as presumptive E. coli was confirmed by a purple colony

104 phenotype on CHROMagar Orientation Medium (CHROMagar, Paris, France) and a positive

105 indole test. Multiple E. coli-positive isolates from each sample were tested by GTG $_{5}$ PCR

106 (Mohapatra, Broersma \& Mazumder, 2007) to minimize the collection of identical genotypes.

107 Isolates were stored using the Microbank Bacterial and Fungal Preservation system (Pro-Lab

108 Diagnostics, Richmond Hill, Canada) at $-80^{\circ} \mathrm{C}$.

109

110 Antimicrobial resistance screening. Isolates were screened for resistance against a panel of

1118 antibiotics (gentamicin, ampicillin, trimethoprim, chloramphenicol, nitrofurantoin, cefpodoxime,

112 meropenem and ciprofloxacin) by the disk diffusion method, according to EUCAST guidelines 
113 (https://www.eucast.org/ast_of_bacteria). They were additionally subjected to a minimum

114 inhibitory concentration assay for colistin by broth dilution, according to EUCAST guidelines.

116 DNA extraction and genome sequencing. A total of 100 isolates were submitted for whole

117 genome sequencing, comprised of multiple isolates per fecal sample from the first three months

118 and one isolate per sample for the remaining two months (Table S1). Genomic DNA was

119 extracted from overnight $1 \mathrm{~mL}$ cultures in Lysogeny Broth (LB; Miller's formulation), maintained

120 at $37^{\circ} \mathrm{C}$ statically in sterile deep-well plates prepared from single colonies taken from overnight

121 Lysogeny Agar plates, according to a previously described 96-well plate lysate method (Foster-

122 Nyarko et al., 2020). Briefly, cultures were pelleted at 3,500 $\times \mathrm{g}$ then resuspended and pre-

123 treated with lysozyme, proteinase $\mathrm{K}$ and RNase A before lysing with $10 \%(\mathrm{w} / \mathrm{v})$ sodium dodecyl

124 sulphate in Tris-EDTA ( $\mathrm{pH}$ 8.0). The DNA was retained on AMPure XP solid-phase reversible

125 immobilization beads (Beckman Coulter, High Wycombe, UK), eluted in Tris/HCl, $\mathrm{pH} 8.0$ and quantified using the Qubit high-sensitivity double-stranded DNA assay (Invitrogen, MA, USA).

127

For sequencing, samples were normalized to $0.5 \mathrm{ng} \cdot \mathrm{\mu L}^{-1}$ with $10 \mathrm{mM}$ Tris- $\mathrm{HCl}$ prior to library preparation, which was carried out with a modified Nextera XT DNA protocol (Foster-Nyarko et al., 2020). The pooled libraries were run at a final concentration of $1.8 \mathrm{pM}$ using the Illumina NextSeq 500 platform (Illumina, CA, USA) following the manufacturer's recommended denaturation and loading procedures, which included a 1\% PhiX spike, with 150 bp paired-end reads.

Genome assembly. Raw sequence data were demultiplexed and converted to fastq format by bcl2fastq v.2.20 (Illumina, CA, USA). Reads from multiple lanes were concatenated into single

137 forward and reverse read files then uploaded to Enterobase (www.enterobase.warwick.ac.uk)

138 for automated quality control and assembly via the QAssembly pipeline (Zhou et al., 2020). All 
139

140

141

142

143

144

145

146

147

148

149

150

151

152

153

154

155

156

157

158

159

160

161

162

163

164

assemblies exceeded the minimum quality requirements for Enterobase (genome length between $3.7-6.4 \mathrm{Mbp} ; \mathrm{N}_{50}>20 \mathrm{~kb}$; number of contigs $\leq 800$; proportion of $\mathrm{Ns}<3 \%$; $>70 \%$ of contigs assigned correctly by Kraken 2 (Wood, Lu \& Langmead, 2019)). The final assemblies were downloaded for further analyses, which were all run on the Cloud Infrastructure for Microbial Bioinformatics (Connor et al., 2016).

Phylogenomic analysis. Initial phylogenomic analysis was carried out in Enterobase for in silico serotyping, fimH allele typing (Roer et al., 2017), multi-locus sequence typing according to the Warwick 7-gene scheme and phylotyping (Beghain et al., 2018), and to construct neighborjoining trees with the NINJA algorithm in GrapeTree (Zhou et al., 2018), based on the Hierarchical Clustering of Core Genome MLST (HierCC) scheme (Zhou et al., 2020). HierCC was also used to de-replicate the strain collection by selecting a single representative isolate from every identical cluster of genomes at the $\mathrm{HCO}$ level. Representative isolates were determined according to the quality score-based system employed as part of dRep v2.5.4, reliant on genome completion, contamination and N50 metrics (OIm et al., 2017). The assemblies for the 33 strains involved in clustering were used to construct a core genome alignment using Snippy v.4.3.2 (https://github.com/tseemann/snippy). A matrix of pairwise single nucleotide polymorphisms (SNPs) was then compiled with snp-dists v.0.7.0

(https://github.com/tseemann/snp-dists). E. coli MG1655 (NC_000913.3) was used as the reference.

To confirm the identities and phylogenetic relationships of the final isolate collection, a second core genome alignment was reconstructed, containing all strains. In addition, we collated a panel of reference genomes (Table S2) for every phylogroup of E. coli and all other Escherichia species, including Shigella flexneri, all of which had been isolated from avian hosts (primarily chickens). These reference genomes were downloaded from the NCBI assembly archive and 
165 included in the core genome alignment, which was then used to reconstruct a core SNP 166 phylogenetic tree with Salmonella bongori and Salmonella enterica as outgroups. IQ-TREE 167 v.2.0.3 (Nguyen et al., 2015) was used with 1,000 bootstrap replications for maximum-likelihood 168 inference of phylogenetic relationships with the best fitting model (TVM+F+ASC+R4) 169 automatically selected by ModelFinder (Kalyaanamoorthy et al., 2017). The resulting tree was 170 visualized and combined with other data using iTOL v.5 (Letunic \& Bork, 2021).

172 Analysis of gene and plasmid content. To process shared gene content across our selected 173 genome catalogue, we used the pangenomics pipeline (Delmont \& Eren, 2018) as implemented 174 in anvi'o v.7.0 (Eren et al., 2015) with open reading frames predicted using prodigal v.2.6 (Hyatt 175 et al., 2010) and annotation using the NCBl's Clusters of Orthologous Groups database 176 (Tatusov et al., 2003). Using NCBI BLAST, the similarity between gene pairs was quantified and 177 subsequently the Markov Cluster algorithm determined clusters of homologous genes, with a minbit heuristics threshold of 0.5 to eliminate weak matches and an MCL inflation of 10 for closely related genomes. FastANI (Jain et al., 2018) was applied for the calculation of average nucleotide identity between genomes. The program anvi-display-pan provided an interactive visualization of the pangenome, with imported average nucleotide identity values being depicted as part of this visualization.

ABRicate v.1.0.1 (https://github.com/tseemann/abricate) was used to search assemblies for 185 genes related to antibiotic resistance and virulence and for plasmid replicons by comparison to the NCBI AMRFinderPlus (https://www.ncbi.nlm.nih.gov/bioproject/PRJNA313047), ecoli_VF (https://github.com/phac-nml/ecoli_vf) and PlasmidFinder (Carattoli et al., 2014) databases, respectively. A custom database was prepared for detection of 24 APEC-related virulence genes based on a literature search (File S1). In each case, identification was defined by minimum coverage of $90 \%$ and minimum identity of $80 \%$ of the respective nucleotide 
191 192 193 194 195 196 201 (Nicoletti et al., 1988).

202

203

204

205

206

207

208

209

210

211

212

213 214 representative (Table S3).

\section{Results}

sequences. To detect plasmid-encoded virulence-associated genes we first identified contigs derived from plasmids using platon v.1.6 in accuracy mode (Schwengers et al., 2020), then searched those contigs against our custom database using ABRicate as described above.

High phylogenomic diversity of isolates recovered. One hundred isolates provisionally identified as $E$. coli were collected from healthy layer hens over a five-month period. An indication of the diversity of Escherichia recovered was provided by the isolation of $1-7$ isolates from up to 6 phylogroups per fecal sample (Table 1). When cultured on MacConkey agar, 15 isolates were non-lactose-fermenters but positive for other E. coli-specific attributes

Following short read whole genome shotgun sequencing and assembly, the HierCC feature within Enterobase identified 33 out of the 100 isolates as belonging to one of 14 clusters at the $\mathrm{HCO}$ level, meaning that the members of each cluster are indistinguishable from each other at every core genome locus interrogated (Table 2). The $14 \mathrm{HCO}$ clusters all contained isolates taken from different fecal samples, spanning the duration of the study, except cluster $\mathrm{HC0}: 148574$, which contained three isolates that all came from a single sample taken in September 2018. A core genome alignment of the 33 strains involved in clustering was used to construct a matrix of pairwise SNP distances, confirming the close relationships between clustered strains, with $2-257$ SNPs detected (Table 2, Table 3). Since we were primarily interested in assessing species diversity within our samples, we therefore removed 19 isolates from the analysis by keeping only the best quality assembly from each $\mathrm{HCO}$ cluster as a

215 
216 In silico evaluation of Warwick 7-gene MLSTs for the remaining 81 isolates revealed the

217 presence of 45 different sequence types, of which 30 were represented by a single isolate each.

218 ST48 (9 isolates), ST10 (7 isolates) and ST2456 (5 isolates) were the most numerous. HC0

219 isolate clusters correlated with MLSTs, supporting the close relationship between strains in each

220 cluster (Table S1). Predicted phylogroups also suggested considerable genetic diversity among

221 the isolates, with at least one representative from all seven E. coli phylogroups except B2, every

222 'cryptic clade' except clade I, and five E. fergusonii (Table 1, Fig. S1). In silico prediction of O:H

223 serotype and fimH alleles also pointed towards considerable diversity, with 30 different $\mathrm{O}$

224 antigens, 22 different $\mathrm{H}$ antigens and 24 different fimH alleles detected (Table S1). Isolates

225 outside of $E$. coli sensu stricto had increased incidence of 'undetermined' results for $O$ antigen

226 and $\mathrm{fimH}$, suggesting that current databases do not cover the whole diversity of these features

227 for the genus. The diversity of strains did not have a significant temporal component as isolates

228 collected in different months were evenly distributed throughout a neighbor-joining phylogenetic

229 tree based on the HierCC results (Fig. S1).

230

231 A core SNP maximum likelihood tree containing the 81 isolates and reference genomes for

232 every clade of Escherichia was in good agreement with the neighbor-joining tree, and the

233 predicted phylogroups clustered with their respective reference genomes (Fig. 2). This validates

234 the reference genome collection, indicating its value for providing a scaffold phylogeny for future

235 studies. Three isolates that were identified as 'group E or clade I' by in silico phylotyping were

236 assigned to phylogroup D based on the core SNP alignment. Isolate Surrey070 was also

237 reassigned, as it clustered more closely with phylogroup $E$ than its predicted phylogroup of D.

239 The pangenome of isolates reflects phylogenomic diversity. More than 500,000 genes

240 were identified within the 81 study isolates and 33 reference genomes before organization into

24114,421 gene clusters, formed by grouping homologous genes according to amino acid similarity, 
242 with a minbit heuristics threshold of 0.5 and an MCL inflation of 10 . The core genome (genes

243 present in all genomes) contained 2,449 gene clusters and the accessory genome (genes

244 present in $>1$ but not all genomes) contained 8,331 gene clusters, while 3,641 singleton clusters

245 (present in only one genome) were identified. All accessory and core genes were hierarchically

246 clustered according to distribution pattern (Fig. 3). Functional hits were assigned to gene

247 clusters according to NCBls Clusters of Orthologous Groups database, with 2,766 unique

248 groups annotated (Table S4). 94\% of core gene clusters were annotated compared to the

249 considerably lower proportion annotated for both accessory (50\%) and singleton (30\%) gene

250 clusters. Functional pathways assigned to annotated gene clusters were primarily associated

251 with translation, ribosomal structure, and biogenesis (9.5\% of annotated core gene clusters)

252 with accessory gene clusters associated with prophages and transposons $(9.8 \%$ of annotated

253 accessory gene clusters). Both core and accessory splits had prominent proportions of gene

254 clusters annotated with carbohydrate transport and metabolism functions $(7.2 \%$ and $10.1 \%$ of

255 annotated gene clusters, respectively).

256

257 Plasmid incompatibility groups are shared throughout Escherichia. A total of 25 different

258 plasmid replicons were identified by comparison with the PlasmidFinder database (Table S5).

259 Eight strains did not contain a plasmid replicon, while two strains (Surrey037 and Surre074)

260 contained 7 different replicons (Fig. 4). The overall rate of plasmid carriage was low, with a

261 mode of 1 replicon and median of 2 . There were 14 instances of an isolate containing multiple

262 replicons of the same identity, although these were confined to the Col(MG828)_1, Col440I_1

263 and ColRNAI_1 groups. The most common replicons were ColRNAI_1 (46 replicons in 35

264 isolates) and p0111_1 (36 replicons in 36 isolates). Of the broad plasmid incompatibility groups,

265 Col plasmids were most frequently found (101 replicons), partly because of their propensity for

266 multiple replicons per isolate, with IncF (58 replicons) and p0111_1 (36 replicons) also highly

267 represented (Fig. 5). There were no clear associations between plasmid incompatibility groups 
268

269

270

271 Overall low virulence potential amongst isolates. To assess the potential for our isolates to

272 cause disease in chickens we looked for genomes containing $\geq 4$ of the virulence-associated

273 genes iutA, $h l y F$, iss, iroN and ompT. This panel has been proposed to identify APEC more

274 accurately than classical serotyping methods (Johnson et al., 2008). Within our isolates,

275 Surrey013, Surrey017 and Surrey034 carried all five genes, while Surrey010 and Surrey050

276 carried all except iutA. However, genes are more strongly correlated with pathogenicity if they

277 are carried on a plasmid rather than the chromosome (Johnson et al., 2008). Both Surrey013

278 and Surrey034 had plasmid-encoded copies of four of the genes, qualifying them as APEC.

279 Surrey017 and Surrey050 carried four plasmid-encoded virulence-associated genes each, but

280 not all were from the panel of five genes so are not counted as APEC under these criteria

281 (Table S6). A further 16 strains carried one or two virulence-associated genes on plasmids.

282

283

284

285

286

287

288

289

290

291

292

293

An alternative diagnostic strategy defines APEC by categorization into any of four associations of virulence (Schouler et al., 2012). By these criteria 5/81 strains are categorized as APEC: Surrey034 (iutA+, $\mathrm{P}(\mathrm{F} 11)-$, frz orf $\left.^{+}\right)$, Surrey001, Surrey002, Surrey014 and Surrey015 (iutA-, sitA+, aec26+). Surrey013 and Surrey017 might additionally be considered marginal as they were only discounted due to their predicted 08 serotype, rather than 078 . Therefore, the two diagnostic measures combined indicate that 6/81 strains are APEC and 3/81 have increased virulence potential but fall short of the cut-off. Overall, only a minority of our isolates are likely to be APEC according to their complement of genetic virulence determinants.

APEC fall into the wider pathotype of Extraintestinal Pathogenic E. coli (ExPEC), which includes named groups causing disease in humans: Neonatal Meningitis E. coli (NMEC), Sepsis- 
294 Associated E. coli (SEPEC) and Uropathogenic E. coli (UPEC) (Sarowska et al., 2019). APEC 295 may be closely related to human ExPEC and may act as a reservoir for ExPEC virulence296 associated genes (Cummins et al., 2019). Therefore, to assess the wider pathogenicity potential 297 of our strains, we compiled a panel of 24 virulence-associated genes. These included the 298 APEC-associated genes above and other typical ExPEC virulence-associated genes (Table 4).

299 Sixteen of these genes were present in at least 1 isolate (Fig. 5). Three isolates (Surrey031, 300 Surrey045 and Surrey062) contained none of the genes and Surrey013 contained the most with 301 14/24 detected. The modal isolate contained 2 genes from the panel, and the median contained 3023 genes.

303

304 Other commonly identified virulence-associated genes from comparison with the ecoli_VF 305 database (a much larger database covering E. coli in general) included fimA - I, yag $V-Z$, 306 ompA, entA - F/S, fepA - D/G and fes. Genes associated with the general secretory pathway $307(g s p C-M)($ Francetic \& Pugsley, 1996) and type III secretion systems 1 and 2 (espL, R, X, Y; 308 eivA, C, E-G, I, J) were also frequently identified (Franzin \& Sircili, 2015; Fox et al., 2020). Many of these genes are associated with intestinal infections of E. coli. Full predicted virulenceassociated gene content of all strains, from comparison with the ecoli_VF database are available in Table S7.

313 Low carriage of predicted antimicrobial resistance genes. From our comparison with the

314 NCBI AMRFinderPlus database, we predicted low levels of antimicrobial resistance. Among the 315 isolates, 62/81 had no predicted resistance genes (Table S8). However, five isolates carried 3 316 predicted resistance genes, one carried 2 genes, and thirteen carried 1 gene. Fourteen isolates 317 carried a gene for predicted tetracycline resistance, nine carried a predicted aminoglycoside 318 resistance gene, three carried a predicted $\beta$-lactamase class bla-TEM gene, two carried a 319 predicted trimethoprim resistance gene, and predicted genes for fosfomycin and sulfonamide 
320

321

322

323

324

325

326

327

328

329

330

331

332

333

334

335

336

337

338

339

340

341

342 The generally low carriage of virulence-associated genes within the isolates suggests that the

343 majority are non-pathogenic and more likely represent commensal members of the gut

344 microbiota (Wigley, 2015). By extension, this also supports the idea that although many strains

345 are capable of opportunistic infection, most frank pathogens in the Escherichia genus belong to

resistance were found in a single isolate each (Fig. 5). Every isolate except three carried a single copy of a $\beta$-lactamase class blaEC gene (one of blaEC, blaEC $8, b l a E C_{13}, b_{1 a E C_{15}}, b / a E C_{18}$ or blaEC $\left.C_{19}\right)$. These genes are frequently detected in genomes of $\beta$-lactam-susceptible isolates and therefore were discounted from the analysis. We did not experimentally verify the predicted resistance phenotypes; however, a preliminary screening following colony isolation also revealed low levels of phenotypic resistance to 9 antibiotics (Table S1).

\section{Discussion}

Our data reveal remarkable diversity within the genus Escherichia from such a commonplace setting and small sample size. After minimal selection during sample collection, we were able to recapitulate almost the entire phylogeny of Escherichia. However, our initial selection for E. coli identity may still have excluded some isolates with phenotypes that differ from typical $E$. coli. On the other hand, the isolation of so many other Escherichia species raises doubts over the specificity of CHROMagar Orientation Medium for Escherichia coli sensu stricto. This finding may have implications for the use of CHROMagar Orientation in clinical research and diagnostics, and warrants further investigation into the growth and morphology of different members of the Escherichia genus on this medium. We confirmed the diversity and identity of the isolates by several different methods, including PCR, in silico phylotyping and MLST, and core genome analysis, with all methods confirming marked diversity. Our final core genomederived phylogenetic tree was also in agreement with previously published phylogenies for the genus (Zhou et al., 2020; Abram et al., 2021; Gilroy et al., 2021). 
346 relatively well-defined pathotypes (Johnson et al., 2008). In support of this conclusion, three key

347 phylogroups, B2, G and clade I, which have been associated with virulence in poultry

348 (Papouskova et al., 2020; Mehat, van Vliet \& La Ragione, 2021), were missing from our

349 collection. This could be due to the relatively small sample size and small flock or might reflect

350 that we only sampled healthy birds. Equally, the most common phylogroup in this study was

351 phylogroup A, which has been linked to commensalism in chickens and other omnivores (Smati

352 et al., 2015). E. albertii and Shigella were also absent from our collection. They are less well

353 studied in birds, but have also both been linked to virulence in poultry (Shi et al., 2014; Gomes

354 et al., 2020). We cannot rule out the possibility that APEC strains were simply lower in

355 abundance and so were overlooked during culturing. As we only sampled healthy birds, it

356 seems likely that this is a true reflection of the prevalence of these phylogroups among our

357 study population. The limitations of our study prevent us from inferring any general conclusions

358 about the distribution of Escherichia strains within chickens in other settings. Nevertheless, if

359 such striking diversity is present in a few birds in a single location, it surely suggests that similar

360 diversity (although perhaps with different population structures) has so far been overlooked

361 elsewhere.

362

363 Although we did not track individual birds temporally and so cannot verify stable colonization of 364 isolates within birds, most fecal samples yielded multiple distinct isolates. There were no clear 365 indications of niche adaptation based on the pangenome and plasmid content of the strains, as 366 gene clusters and plasmid replicons were spread fairly evenly among phylogenomic groups, 367 and most phylogroups contained few isolates. The use of only short-read data also limited the 368 completeness of our assemblies and our ability to identify plasmids and plasmid-encoded genes 369 (Arredondo-Alonso et al., 2017). Further work involving more isolates and using both short- and 370 long-read data will be necessary to identify unique, characteristic genetic components for each 
371 species and phylogroup, and to establish their spatial and functional niches within the chicken 372 gut.

373

374 Recent studies have found similarly diverse populations of Escherichia after sampling of healthy 375 pigs (Ahmed, Olsen \& Herrero-Fresno, 2017; Bok et al., 2020), cattle and sheep (Shaw et al., 376 2021), and non-human primates (Foster-Nyarko et al., 2020), although it is notable that

377 representatives of the 'cryptic clades' are scarce within these data. The reasons for this scarcity 378 are unclear but are likely to include the use of different selection methods and the focus on 379 virulent/AMR strains and E. coli sensu stricto. Escherichia diversity varies among samples from 380 healthy humans (Bok et al., 2018; Foster-Nyarko et al., 2021a). High Escherichia species 381 diversity has been detected within chickens previously (Vounba et al., 2019a; Foster-Nyarko et 382 al., 2021b), although not from such a narrow pool of host birds as in the current study. Members of the cryptic clades have also previously been found in relatively high abundance in wild birds of various species (Clermont et al., 2011) but a definitive link to chickens has not been established. A recent metagenomics study included 60 different fecal samples from the same birds used here. Culturing of 3 of those samples yielded isolates of E. marmotae, E. whittamii and phylogroups A, B1, C, D and E, reflecting the diversity found in this study (Gilroy et al., 2021). However, most studies in chickens have taken samples from diseased birds and/or have focused on antimicrobial resistance and pathogenicity of the isolates recovered (Braga et al., 2016; Cummins et al., 2019; Vounba et al., 2019b; Papouskova et al., 2020; Kubelová et al., 391 2021).

392

\section{Conclusions}

394 The dearth of whole-genome sequencing studies that include all members of Escherichia (and 395 sufficient metadata) skews our understanding of the genus and makes it impossible to discern 
397 398

and so on. Consequently, there is much still to learn about the population structure and ecology of Escherichia (Lagerstrom \& Hadly, 2021). We were surprised to find that isolates representing almost the entire known phylogeny of Escherichia were recovered from fecal samples from a small flock of healthy layer chickens, including species that have only recently been recognized (previously known as cryptic clades) and for which chicken-associated isolates have not been widely reported. These isolates had low carriage rates of antimicrobial resistance genes and virulence factors, suggesting that similar isolates might often be overlooked in studies that focus on these traits. Our findings highlight the surprising diversity of Escherichia harbored by even an individual chicken and emphasize the need to broaden the focus of research to encapsulate the full variety of species.

\section{Acknowledgements}

We thank Mr Steven Rudder and Mr David Baker for their assistance with sequencing, and the farm for assisting with the study.

\section{References}

Abram K, Udaondo Z, Bleker C, Wanchai V, Wassenaar TM, Robeson MS, Ussery DW. 2021. Mash-based analyses of Escherichia coli genomes reveal 14 distinct phylogroups. Communications Biology 4. DOI: 10.1038/s42003-020-01626-5.

Ahmed S, Olsen JE, Herrero-Fresno A. 2017. The genetic diversity of commensal Escherichia coli strains isolated from nonantimicrobial treated pigs varies according to age group. PLoS ONE 12. DOI: 10.1371/journal.pone.0178623.

Arredondo-Alonso S, Willems RJ, van Schaik W, Schürch AC. 2017. On the (im)possibility of reconstructing plasmids from whole-genome short-read sequencing data. Microbial Genomics 3. DOI: 10.1099/mgen.0.000128.

Beghain J, Bridier-Nahmias A, Nagard H Le, Denamur E, Clermont O. 2018. ClermonTyping: An easy-to-use and accurate in silico method for Escherichia genus strain phylotyping. Microbial Genomics 4:1-8. DOI: 10.1099/mgen.0.000192.

Bok E, Kożańska A, Mazurek-Popczyk J, Wojciech M, Baldy-Chudzik K. 2020. Extended phylogeny and extraintestinal virulence potential of commensal escherichia coli from piglets and sows. International Journal of Environmental Research and Public Health 17. DOI: 10.3390/ijerph17010366.

Bok E, Mazurek J, Myc A, Stosik M, Wojciech M, Baldy-Chudzik K. 2018. Comparison of commensal escherichia coli isolates from adults and young children in Lubuskie province, Poland: Virulence potential, phylogeny and antimicrobial resistance. International Journal 
432

433

434

435

436

437

438

439

440

441

442

443

444

445

446

447

448

449

450

451

452

453

454

455

456

457

458

459

460

461

462

463

464

465

466

467

468

469

470

471

472

473

474

475

476

477

of Environmental Research and Public Health 15:1-19. DOI: 10.3390/ijerph15040617.

Braga JFV, Chanteloup NK, Trotereau A, Baucheron S, Guabiraba R, Ecco R, Schouler C. 2016. Diversity of Escherichia coli strains involved in vertebral osteomyelitis and arthritis in broilers in Brazil. BMC Veterinary Research 12:1-12. DOI: 10.1186/s12917-016-0762-0.

Carattoli A, Zankari E, Garciá-Fernández A, Larsen MV, Lund O, Villa L, Aarestrup FM, Hasman H. 2014. In Silico detection and typing of plasmids using plasmidfinder and plasmid multilocus sequence typing. Antimicrobial Agents and Chemotherapy 58:38953903. DOI: 10.1128/AAC.02412-14.

Clermont O, Christenson JK, Denamur E, Gordon DM. 2013. The Clermont Escherichia coli phylo-typing method revisited: Improvement of specificity and detection of new phylogroups. Environmental Microbiology Reports 5:58-65. DOI: 10.1111/1758-2229.12019.

Clermont O, Dixit OVA, Vangchhia B, Condamine B, Dion S, Bridier-Nahmias A, Denamur E, Gordon D. 2019. Characterization and rapid identification of phylogroup G in Escherichia coli, a lineage with high virulence and antibiotic resistance potential. Environmental Microbiology 21:3107-3117. DOI: 10.1111/1462-2920.14713.

Clermont O, Gordon DM, Brisse S, Walk ST, Denamur E. 2011. Characterization of the cryptic Escherichia lineages: Rapid identification and prevalence. Environmental Microbiology 13:2468-2477. DOI: 10.1111/j.1462-2920.2011.02519.x.

Connor TR, Loman NJ, Thompson S, Smith A, Southgate J, Poplawski R, Bull MJ, Richardson E, Ismail M, Thompson SE, Kitchen C, Guest M, Bakke M, Sheppard SK, Pallen MJ. 2016. CLIMB (the Cloud Infrastructure for Microbial Bioinformatics): an online resource for the medical microbiology community. Microbial genomics 2:e000086. DOI: 10.1099/mgen.0.000086.

Cummins ML, Reid CJ, Chowdhury PR, Bushell RN, Esbert N, Tivendale KA, Noormohammadi AH, Islam S, Marenda MS, Browning GF, Markham PF, Djordjevic SP. 2019. Whole genome sequence analysis of Australian avian pathogenic Escherichia coli that carry the class 1 integrase gene. Microbial Genomics 5. DOI: 10.1099/mgen.0.000250.

Delmont TO, Eren EM. 2018. Linking pangenomes and metagenomes: The Prochlorococcus metapangenome. PeerJ 2018:1-23. DOI: 10.7717/peerj.4320.

Devanga Ragupathi NK, Muthuirulandi Sethuvel DP, Inbanathan FY, Veeraraghavan B. 2018. Accurate differentiation of Escherichia coli and Shigella serogroups: challenges and strategies. New Microbes and New Infections 21:58-62. DOI: 10.1016/j.nmni.2017.09.003.

Dziva F, Stevens MP. 2008. Colibacillosis in poultry: Unravelling the molecular basis of virulence of avian pathogenic Escherichia coli in their natural hosts. Avian Pathology 37:355-366. DOI: 10.1080/03079450802216652.

Eren AM, Esen OC, Quince C, Vineis JH, Morrison HG, Sogin ML, Delmont TO. 2015. Anvi'o: An advanced analysis and visualization platformfor 'omics data. PeerJ 2015:1-29. DOI: 10.7717/peerj.1319.

Foster-Nyarko E, Alikhan N, Ikumapayi UN, Sarwar G, Okoi C, Tientcheu PM, Defernez M, O’Grady J, Antonio M, Pallen MJ. 2021a. Genomic diversity of Escherichia coli from healthy children in rural Gambia. PeerJ 9:e10572. DOI: 10.7717/peerj.10572.

Foster-Nyarko E, Alikhan NF, Ravi A, Thilliez G, Thomson NM, Baker D, Kay G, Cramer JD, O'grady J, Antonio M, Pallen MJ. 2020. Genomic diversity of Escherichia coli isolates from non-human primates in the Gambia. Microbial Genomics 6:1-15. DOI: 10.1099/mgen.0.000428.

Foster-Nyarko E, Alikhan N-F, Ravi A, Thomson NM, Jarju S, Kwambana-Adams BA, Secka A, 
478

479

480

481

482

483

484

485

486

487

488

489

490

491

492

493

494

495

496

497

498

499

500

501

502

503

504

505

506

507

508

509

510

511

512

513

514

515

516

517

518

519

520

521

522

523
O’grady J, Antonio M, Pallen MJ. 2021b. Genomic diversity of escherichia coli isolates from backyard chickens and guinea fowl in the gambia. Microbial Genomics 7. DOI: 10.1099/mgen.0.000484.

Fox S, Goswami C, Holden M, Connolly JPR, Mordue J, O’Boyle N, Roe A, Connor M, Leanord A, Evans TJ. 2020. A highly conserved complete accessory Escherichia coli type III secretion system 2 is widespread in bloodstream isolates of the ST69 lineage. Scientific Reports 10:1-11. DOI: 10.1038/s41598-020-61026-X.

Francetic O, Pugsley AP. 1996. The cryptic general secretory pathway (gsp) operon of Escherichia coli K-12 encodes functional proteins. Journal of Bacteriology 178:3544-3549. DOI: $10.1128 /$ jb.178.12.3544-3549.1996.

Franzin FM, Sircili MP. 2015. Locus of enterocyte effacement: A pathogenicity island involved in the virulence of enteropathogenic and enterohemorragic escherichia coli subjected to a complex network of gene regulation. BioMed Research International 2015. DOI: $10.1155 / 2015 / 534738$.

Fratamico PM, DebRoy C, Liu Y, Needleman DS, Baranzoni GM, Feng P. 2016. Advances in molecular serotyping and subtyping of Escherichia coli. Frontiers in Microbiology 7:1-8. DOI: $10.3389 /$ fmicb.2016.00644.

Gilroy R, Ravi A, Getino M, Pursley I, Horton DL, Alikhan NF, Baker D, Gharbi K, Hall N, Watson M, Adriaenssens EM, Foster-Nyarko E, Jarju S, Secka A, Antonio M, Oren A, Chaudhuri RR, Ragione R La, Hildebrand F, Pallen MJ. 2021. Extensive microbial diversity within the chicken gut microbiome revealed by metagenomics and culture. PeerJ 9:1-142. DOI: $10.7717 /$ peerj.10941.

Gomes TAT, Ooka T, Hernandes RT, Yamamoto D, Hayashi T. 2020. Escherichia albertii Pathogenesis. EcoSal Plus 9. DOI: 10.1128/ecosalplus.esp-0015-2019.

Hyatt D, Chen GL, LoCascio PF, Land ML, Larimer FW, Hauser LJ. 2010. Prodigal: Prokaryotic gene recognition and translation initiation site identification. $B M C$ Bioinformatics 11. DOI: 10.1186/1471-2105-11-119.

Jain C, Rodriguez-R LM, Phillippy AM, Konstantinidis KT, Aluru S. 2018. High throughput ANI analysis of $90 \mathrm{~K}$ prokaryotic genomes reveals clear species boundaries. Nature Communications 9:1-8. DOI: 10.1038/s41467-018-07641-9.

Jang J, Hur HG, Sadowsky MJ, Byappanahalli MN, Yan T, Ishii S. 2017. Environmental Escherichia coli: ecology and public health implications - a review. Journal of Applied Microbiology 123:570-581. DOI: 10.1111/jam.13468.

Johnson TJ, Wannemuehler Y, Doetkott C, Johnson SJ, Rosenberger SC, Nolan LK. 2008. Identification of minimal predictors of avian pathogenic Escherichia coli virulence for use as a rapid diagnostic tool. Journal of Clinical Microbiology 46:3987-3996. DOI: 10.1128/JCM.00816-08.

Kalyaanamoorthy S, Minh BQ, Wong TKF, Von Haeseler A, Jermiin LS. 2017. ModelFinder: Fast model selection for accurate phylogenetic estimates. Nature Methods 14:587-589. DOI: $10.1038 /$ nmeth.4285.

Kaper JB, Nataro JP, Mobley HLT. 2004. Pathogenic Escherichia coli. Nature Reviews Microbiology 2:123-140. DOI: 10.1038/nrmicro818.

Konstantinidis KT, Tiedje JM. 2005. Genomic insights that advance the species definition for prokaryotes. Proceedings of the National Academy of Sciences of the United States of America 102:2567-72. DOI: 10.1073/pnas.0409727102.

Kubelová M, Koláčková I, Karpíšková R, Gelbíčová T, Florianová M, Kalová A. 2021. 
524

525

526

527

528

529

530

531

532

533

534

535

536

537

538

539

540

541

542

543

544

545

546

547

548

549

550

551

552

553

554

555

556

557

558

559

560

561

562

563

564

565

566

567

568

569

Virulence properties of mcr-1-positive escherichia coli isolated from retail poultry meat. Microorganisms 9:1-9. DOI: 10.3390/microorganisms9020308.

Lagerstrom KM, Hadly EA. 2021. The under-investigated wild side of Escherichia coli: Genetic diversity, pathogenicity and antimicrobial resistance in wild animals. Proceedings of the Royal Society B: Biological Sciences 288. DOI: 10.1098/rspb.2021.0399.

Letunic I, Bork P. 2021. Interactive tree of life (iTOL) v5: An online tool for phylogenetic tree display and annotation. Nucleic Acids Research 49:W293-W296. DOI: 10.1093/nar/gkab301.

Liu S, Jin D, Lan R, Wang Y, Meng Q, Dai H, Lu S, Hu S, Xu J. 2015. Escherichia marmotae sp. nov., isolated from faeces of Marmota himalayana. International Journal of Systematic and Evolutionary Microbiology 65:2130-2134. DOI: 10.1099/ijs.0.000228.

Lu S, Jin D, Wu S, Yang J, Lan R, Bai X, Liu S, Meng Q, Yuan X, Zhou J, Pu J, Chen Q, Dai H, $\mathrm{Hu}$ Y, Xiong Y, Ye C, Xu J. 2016. Insights into the evolution of pathogenicity of Escherichia coli from genomic analysis of intestinal E. coli of Marmota himalayana in Qinghai-Tibet plateau of China. Emerging Microbes and Infections 5. DOI: 10.1038/emi.2016.122.

Maiden MCJ, Bygraves JA, Feil E, Morelli G, Russell JE, Urwin R, Zhang Q, Zhou J, Zurth K, Caugant DA, Feavers IM, Achtman M, Spratt BG. 1998. Multilocus sequence typing: A portable approach to the identification of clones within populations of pathogenic microorganisms. Proceedings of the National Academy of Sciences of the United States of America 95:3140-3145. DOI: 10.1073/pnas.95.6.3140.

Mehat JW, van Vliet AHM, La Ragione RM. 2021. The Avian Pathogenic Escherichia coli (APEC) pathotype is comprised of multiple distinct, independent genotypes. Avian Pathology 50:402-416. DOI: 10.1080/03079457.2021.1915960.

Mohapatra BR, Broersma K, Mazumder A. 2007. Comparison of five rep-PCR genomic fingerprinting methods for differentiation of fecal Escherichia coli from humans, poultry and wild birds. FEMS Microbiology Letters 277:98-106. DOI: 10.1111/j.15746968.2007.00948.x.

Nguyen LT, Schmidt HA, Von Haeseler A, Minh BQ. 2015. IQ-TREE: A fast and effective stochastic algorithm for estimating maximum-likelihood phylogenies. Molecular Biology and Evolution 32:268-274. DOI: 10.1093/molbev/msu300.

Nicoletti M, Superti F, Conti C, Calconi A, Zagaglia C. 1988. Virulence factors of lactosenegative Escherichia coli strains isolated from children with diarrhea in Somalia. Journal of Clinical Microbiology 26:524-529. DOI: 10.1128/jcm.26.3.524-529.1988.

Olm MR, Brown CT, Brooks B, Banfield JF. 2017. DRep: A tool for fast and accurate genomic comparisons that enables improved genome recovery from metagenomes through dereplication. ISME Journal 11:2864-2868. DOI: 10.1038/ismej.2017.126.

Papouskova A, Papouskova A, Masarikova M, Masarikova M, Valcek A, Valcek A, Senk D, Cejkova D, Jahodarova E, Cizek A, Cizek A. 2020. Genomic analysis of Escherichia coli strains isolated from diseased chicken in the Czech Republic. BMC Veterinary Research 16:1-10. DOI: 10.1186/s12917-020-02407-2.

Priest FG, Barker M. 2010. Gram-negative bacteria associated with brewery yeasts: Reclassification of Obesumbacterium proteus biogroup 2 as Shimwellia pseudoproteus gen. nov., sp. nov., and transfer of Escherichia blattae to Shimwellia blattae comb. nov. International Journal of Systematic and Evolutionary Microbiology 60:828-833. DOI: 10.1099/ijs.0.013458-0. 
570

571

572

573

574

575

576

577

578

579

580

581

582

583

584

585

586

587

588

589

590

591

592

593

594

595

596

597

598

599

600

601

602

603

604

605

606

607

608

609

610

611

612

613

614

615

van der Putten BCL, Matamoros S, Mende DR, Scholl ER, Consortium† C, Schultsz C. 2021. Escherichia ruysiae sp. nov., a novel Gram-stain-negative bacterium, isolated from a faecal sample of an international traveller. International Journal of Systematic and Evolutionary Microbiology 71. DOI: 10.1099/ijsem.0.004609.

Roer L, Tchesnokova V, Allesøe R, Muradova M, Chattopadhyay S, Ahrenfeldt J, Thomsen MCF, Lund O, Hansen F, Hammerum AM, Sokurenko E, Hasman H. 2017. Development of a Web Tool for Escherichia coli Subtyping Based on fimH Alleles. Journal of clinical microbiology 55:2538-2543. DOI: 10.1128/JCM.00737-17.

Sarowska J, Futoma-Koloch B, Jama-Kmiecik A, Frej-Madrzak M, Ksiazczyk M, BuglaPloskonska G, Choroszy-Krol I. 2019. Virulence factors, prevalence and potential transmission of extraintestinal pathogenic Escherichia coli isolated from different sources: Recent reports. Gut Pathogens 11:1-16. DOI: 10.1186/s13099-019-0290-0.

Schouler C, Schaeffer B, Brée A, Mora A, Dahbi G, Biet F, Oswald E, Mainil J, Blanco J, Moulin-Schouleur M. 2012. Diagnostic strategy for identifying avian pathogenic Escherichia coli based on four patterns of virulence genes. Journal of Clinical Microbiology 50:1673-1678. DOI: 10.1128/JCM.05057-11.

Schürch AC, Arredondo-Alonso S, Willems RJL, Goering R V. 2018. Whole genome sequencing options for bacterial strain typing and epidemiologic analysis based on single nucleotide polymorphism versus gene-by-gene-based approaches. Clinical Microbiology and Infection 24:350-354. DOI: 10.1016/j.cmi.2017.12.016.

Schwengers O, Barth P, Falgenhauer L, Hain T, Chakraborty T, Goesmann A. 2020. Platon: Identification and characterization of bacterial plasmid contigs in short-read draft assemblies exploiting protein sequence-based replicon distribution scores. Microbial Genomics 6:1-12. DOI: 10.1099/mgen.0.000398.

Selander RK, Musser JM, Caugant DA, Gilmour MN, Whittam TS. 1987. Population genetics of pathogenic bacteria. Microbial pathogenesis 3:1-7. DOI: 10.1016/0882-4010(87)90032-5.

Shaw LP, Chau KK, Kavanagh J, AbuOun M, Stubberfield E, Gweon HS, Barker L, Rodger G, Bowes MJ, Hubbard ATM, Pickford H, Swann J, Gilson D, Smith RP, Hoosdally SJ, Sebra R, Brett H, Peto TEA, Bailey MJ, Crook DW, Read DS, Anjum MF, Walker AS, Stoesser N. 2021. Niche and local geography shape the pangenome of wastewater- and livestockassociated Enterobacteriaceae. Science Advances 7:eabe3868. DOI: 10.1126/sciadv.abe3868.

Shi R, Yang X, Chen L, Chang HT, Liu HY, Zhao J, Wang XW, Wang CQ. 2014. Pathogenicity of Shigella in chickens. PLoS ONE 9:1-7. DOI: 10.1371/journal.pone.0100264.

Smati M, Clermont O, Bleibtreu A, Fourreau F, David A, Daubié AS, Hignard C, Loison O, Picard B, Denamur E. 2015. Quantitative analysis of commensal Escherichia coli populations reveals host-specific enterotypes at the intra-species level. MicrobiologyOpen 4:604-615. DOI: 10.1002/mbo3.266.

Tatusov RL, Fedorova ND, Jackson JD, Jacobs AR, Kiryutin B, Koonin E V., Krylov DM, Mazumder R, Smirnov S, Nikolskaya AN, Rao BS, Mekhedov SL, Sverlov A V., Vasudevan S, Wolf YI, Yin JJ, Natale DA. 2003. The COG database: An updated vesion includes eukaryotes. BMC Bioinformatics 4:1-14. DOI: 10.1186/1471-2105-4-41.

Tenaillon O, Skurnik D, Picard B, Denamur E. 2010. The population genetics of commensal Escherichia coli. Nature Reviews Microbiology 8:207-217. DOI: 10.1038/nrmicro2298.

Touchon M, Perrin A, De Sousa JAM, Vangchhia B, Burn S, O’Brien CL, Denamur E, Gordon D, Rocha EPC. 2020. Phylogenetic background and habitat drive the genetic diversification 
616

617

618

619

620

621

622

623

624

625

626

627

628

629

630

631

632

633

634

635

636

637

638

639

640

641

642

643

644

of Escherichia coli. DOI: 10.1371/journal.pgen.1008866.

Uelze L, Grützke J, Borowiak M, Hammerl JA, Juraschek K, Deneke C, Tausch SH, Malorny B. 2020. Typing methods based on whole genome sequencing data. One Health Outlook 2:119. DOI: $10.1186 / \mathrm{s} 42522-020-0010-1$.

Vounba P, Arsenault J, Bada-Alambédji R, Fairbrother JM. 2019a. Antimicrobial Resistance and Potential Pathogenicity of Escherichia coli Isolates from Healthy Broilers in Québec, Canada. Microbial Drug Resistance 25:1111-1121. DOI: 10.1089/mdr.2018.0403.

Vounba P, Arsenault J, Bada-Alambédji R, Fairbrother JM. 2019b. Pathogenic potential and the role of clones and plasmids in beta-lactamase-producing E. coli from chicken faeces in Vietnam. BMC Veterinary Research 15:1-13. DOI: 10.1186/s12917-019-1849-1.

Walk ST, Alm EW, Gordon DM, Ram JL, Toranzos GA, Tiedje JM, Whittam TS. 2009. Cryptic lineages of the genus Escherichia. Applied and Environmental Microbiology 75:6534-6544. DOI: 10.1128/AEM.01262-09.

Wigley P. 2015. Blurred lines: Pathogens, commensals, and the healthy gut. Frontiers in Veterinary Science 2:10-13. DOI: 10.3389/fvets.2015.00040.

Wood DE, Lu J, Langmead B. 2019. Improved metagenomic analysis with Kraken 2. Genome biology 20:257. DOI: 10.1186/s13059-019-1891-0.

Yang F, Yang J, Zhang X, Chen L, Jiang Y, Yan Y, Tang X, Wang J, Xiong Z, Dong J, Xue Y, Zhu Y, Xu X, Sun L, Chen S, Nie H, Peng J, Xu J, Wang Y, Yuan Z, Wen Y, Yao Z, Shen Y, Qiang B, Hou Y, Yu J, Jin Q. 2005. Genome dynamics and diversity of Shigella species, the etiologic agents of bacillary dysentery. Nucleic Acids Research 33:6445-6458. DOI: 10.1093/nar/gki954.

Zhou Z, Alikhan NF, Mohamed K, Fan Y, Achtman M. 2020. The EnteroBase user's guide, with case studies on Salmonella transmissions, Yersinia pestis phylogeny, and Escherichia core genomic diversity. Genome Research 30:138-152. DOI: 10.1101/gr.251678.119.

Zhou Z, Alikhan NF, Sergeant MJ, Luhmann N, Vaz C, Francisco AP, Carriço JA, Achtman M. 2018. Grapetree: Visualization of core genomic relationships among 100,000 bacterial pathogens. Genome Research 28:1395-1404. DOI: 10.1101/gr.232397.117.

Peer) reviewing PDF | (2021:11:67677:1:0:NEW 30 Dec 2021) 


\section{Table $\mathbf{1}$ (on next page)}

Collection dates, age of birds and number of isolates recovered for each fecal sample.

Following culturing on MacConkey agar and CHROMagar Orientation medium, 100 presumptive $E$. coli were isolated. Multiple colonies per fecal sample were screened by $\mathrm{GTG}_{5}$ PCR to reduce the collection of identical strains. Whole-genome sequence analysis revealed the large diversity of Escherichia present despite apparently selecting for E. coli, although only 81 of the initial strains were distinguishable at the $\mathrm{HCO}$ level using HierCC clustering (see Materials and Methods). Phylogroups of $E$. coli are referred to by individual letters; cryptic clades are referred to by Roman numerals in parentheses; Ef, E. fergusonii. 


\section{Table 1: Collection dates, age of birds and number of isolates recovered for each fecal}

2 sample.

3 Following culturing on MacConkey agar and CHROMagar Orientation medium, 100

4 presumptive E. coli were isolated. Multiple colonies per fecal sample were screened by GTG5

5 PCR to reduce the collection of identical strains. Whole-genome sequence analysis revealed the

6 large diversity of Escherichia present despite apparently selecting for E. coli, although only 81 of

7 the initial strains were distinguishable at the $\mathrm{HCO}$ level using HierCC clustering (see Materials

8 and Methods). Phylogroups of E. coli are referred to by individual letters; cryptic clades are

9 referred to by Roman numerals in parentheses; Ef, E. fergusonii.

10

\begin{tabular}{llllll}
\hline $\begin{array}{l}\text { Faecal } \\
\text { sample }\end{array}$ & $\begin{array}{l}\text { Date } \\
\text { collected }\end{array}$ & $\begin{array}{l}\text { Age of } \\
\text { birds } \\
\text { (weeks) }\end{array}$ & $\begin{array}{l}\text { GTG } \\
\text { unique } \\
\text { isolates }\end{array}$ & $\begin{array}{l}\text { HCO } \\
\text { unique } \\
\text { isolates }\end{array}$ & Phylogroups \\
\hline \hline 1 & $09 / 07 / 2018$ & 84 & 5 & 5 & A, B1 \\
2 & $09 / 07 / 2018$ & 84 & 6 & 5 & A, B1, (II) \\
3 & $09 / 07 / 2018$ & 84 & 6 & 6 & A, B1, C \\
4 & $09 / 07 / 2018$ & 84 & 6 & 3 & A, Ef, (V) \\
5 & $09 / 07 / 2018$ & 84 & 6 & 4 & A, D, (V) \\
6 & $13 / 08 / 2018$ & 89 & 7 & 7 & A, B1, F, Ef, (III), (V) \\
7 & $13 / 08 / 2018$ & 89 & 5 & 4 & A, B1, D \\
8 & $13 / 08 / 2018$ & 89 & 6 & 5 & A, Ef, (III), (V) \\
9 & $13 / 08 / 2018$ & 89 & 6 & 6 & A, B1, (V) \\
10 & $13 / 08 / 2018$ & 89 & 5 & 3 & A, B1, D, (III), (IV) \\
11 & $10 / 09 / 2018$ & 93 & 6 & 6 & A, B1, E, Ef, (III) \\
12 & $10 / 09 / 2018$ & 93 & 7 & 5 & A, B1, E, Ef \\
13 & $10 / 09 / 2018$ & 93 & 7 & 5 & A, B1, D, (III) \\
14 & $10 / 09 / 2018$ & 93 & 7 & 4 & A, Ef, (IV), (V) \\
15 & $10 / 09 / 2018$ & 93 & 6 & 4 & A, D, F, (III), (V) \\
16 & $08 / 10 / 2018$ & 97 & 1 & 1 & B1 \\
17 & $08 / 10 / 2018$ & 97 & 1 & 1 & (IV) \\
18 & $08 / 10 / 2018$ & 97 & 1 & 1 & (III) \\
19 & $08 / 10 / 2018$ & 97 & 1 & 1 & A \\
20 & $08 / 10 / 2018$ & 97 & 1 & 1 & D \\
21 & $12 / 11 / 2018$ & 102 & 1 & 1 & (V) \\
22 & $12 / 11 / 2018$ & 102 & 1 & 1 & D \\
23 & $12 / 11 / 2018$ & 102 & 1 & 1 & B1 \\
24 & $12 / 11 / 2018$ & 102 & 1 & 1 & A \\
\hline & & & $\mathbf{1 0 0}$ & $\mathbf{8 1}$ & \\
\hline & & & & & \\
\hline
\end{tabular}

11 
Table 2 (on next page)

Clustering of isolates by HierCC level $\mathrm{HCO}$ and selection of representative isolate for each cluster. 
1 Table 2: Clustering of isolates by HierCC level $\mathrm{HCO}$ and selection of representative isolate 2 for each cluster.

3

\begin{tabular}{lllll}
\hline HC0 cluster & MLST & Isolates & $\begin{array}{l}\text { Core SNP } \\
\text { distance }\end{array}$ & $\begin{array}{l}\text { Representative } \\
\text { isolate }\end{array}$ \\
\hline \hline 148525 & 5643 & 021,043 & 124 & 021 \\
148529 & 155 & 007,037 & 21 & 037 \\
148537 & 48 & 024,032 & 11 & 032 \\
148541 & 1112 & 026,067 & 6 & 067 \\
148542 & 1638 & 006,055 & 11 & 006 \\
148547 & 5573 & $025,041,057,073,087$ & See Table 3 & 025 \\
148548 & 1276 & 034,089 & 18 & 034 \\
148555 & 48 & 018,042 & 20 & 042 \\
148556 & 7059 & 045,085 & 55 & 045 \\
148564 & 1844 & $019,050,078$ & See Table 3 & 050 \\
148571 & 6540 & 063,080 & 257 & 063 \\
148574 & 2456 & $068,069,071$ & See Table 3 & 068 \\
148603 & 48 & 004,081 & 2 & 004 \\
148607 & 11513 & 020,058 & 143 & 058 \\
\hline & & & &
\end{tabular}

4 


\section{Table 3(on next page)}

SNP distance matrices for $\mathrm{HCO}$ clusters of $>2$ isolates.

Table 2 provides SNP distances for 11 HCO clusters comprising pairs of isolates. The remaining 3 clusters contained $>2$ isolates, requiring a matrix for full pairwise comparison. 
1 Table 3: SNP distance matrices for HCO clusters of $>2$ isolates.

2 Table 2 provides SNP distances for 11 HCO clusters comprising pairs of isolates. The remaining

33 clusters contained $>2$ isolates, requiring a matrix for full pairwise comparison.

4

\begin{tabular}{c|cccc}
\hline \multicolumn{5}{c}{ SNP distance matrix for Cluster } \\
HC0_148547 \\
\hline Strains & $\mathbf{0 4 1}$ & $\mathbf{0 5 7}$ & $\mathbf{0 7 3}$ & $\mathbf{0 8 7}$ \\
\hline $\mathbf{0 2 5}$ & 9 & 9 & 9 & 4 \\
$\mathbf{0 4 1}$ & 0 & 5 & 11 & 11 \\
$\mathbf{0 5 7}$ & & 0 & 13 & 8 \\
$\mathbf{0 7 3}$ & & & 0 & 10 \\
\hline
\end{tabular}

SNP distance matrix for Cluster HCO_148564

\begin{tabular}{l|cc}
\hline & $\mathbf{0 5 0}$ & $\mathbf{0 7 8}$ \\
\hline $\mathbf{0 1 9}$ & 16 & 15 \\
$\mathbf{0 5 0}$ & 0 & 14 \\
\hline
\end{tabular}

SNP distance matrix for Cluster

\begin{tabular}{l|cc}
\multicolumn{3}{|c}{ HCO_148574 } \\
\hline & 069 & 071 \\
\hline $\mathbf{0 6 8}$ & 8 & 14 \\
$\mathbf{0 6 9}$ & 0 & 10
\end{tabular}

5

6 


\section{Table 4 (on next page)}

Members of the panel of virulence-associated genes used to predict virulence potential of isolates in this study. 
1 Table 4: Members of the panel of virulence-associated genes used to predict virulence 2 potential of isolates in this study.

3

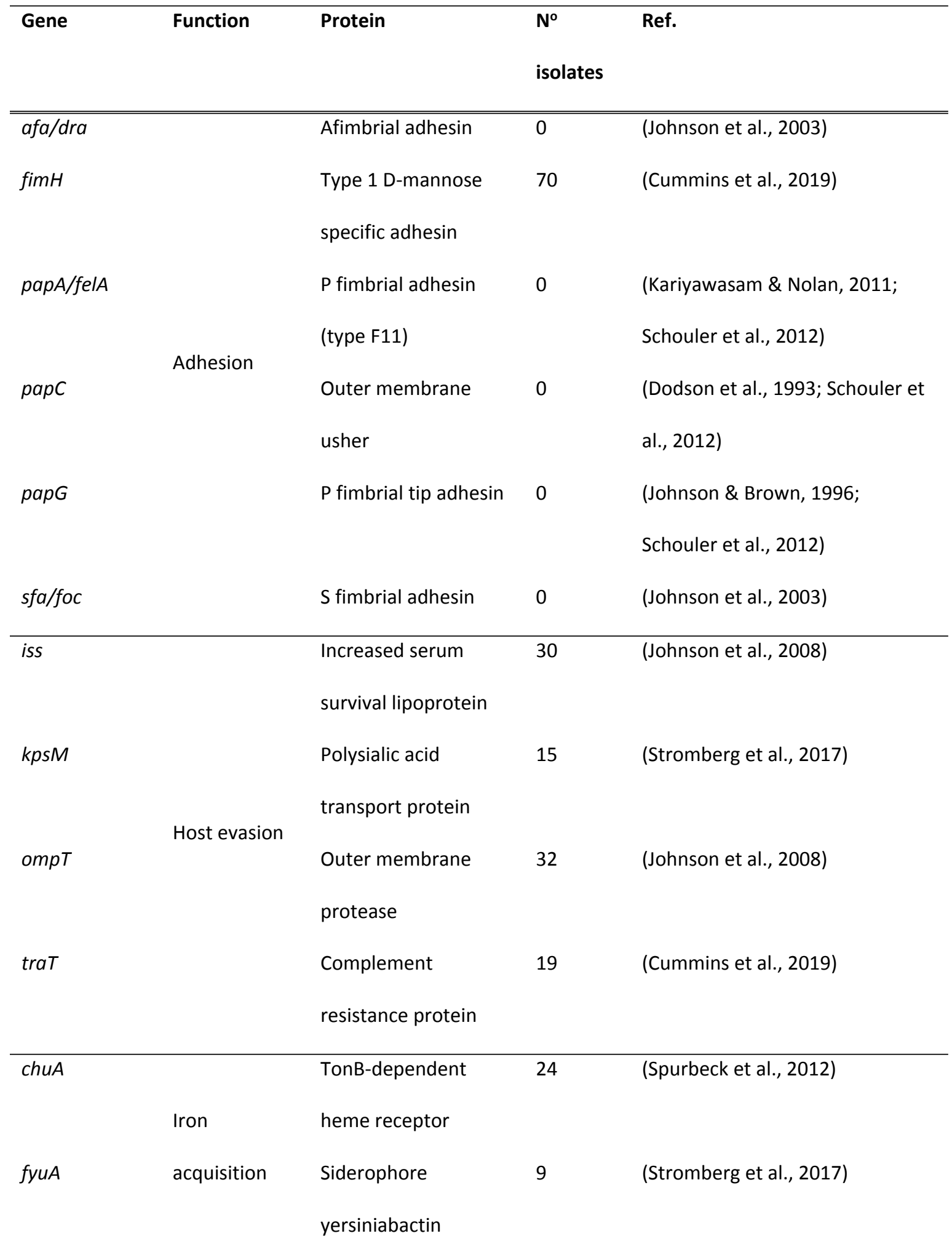




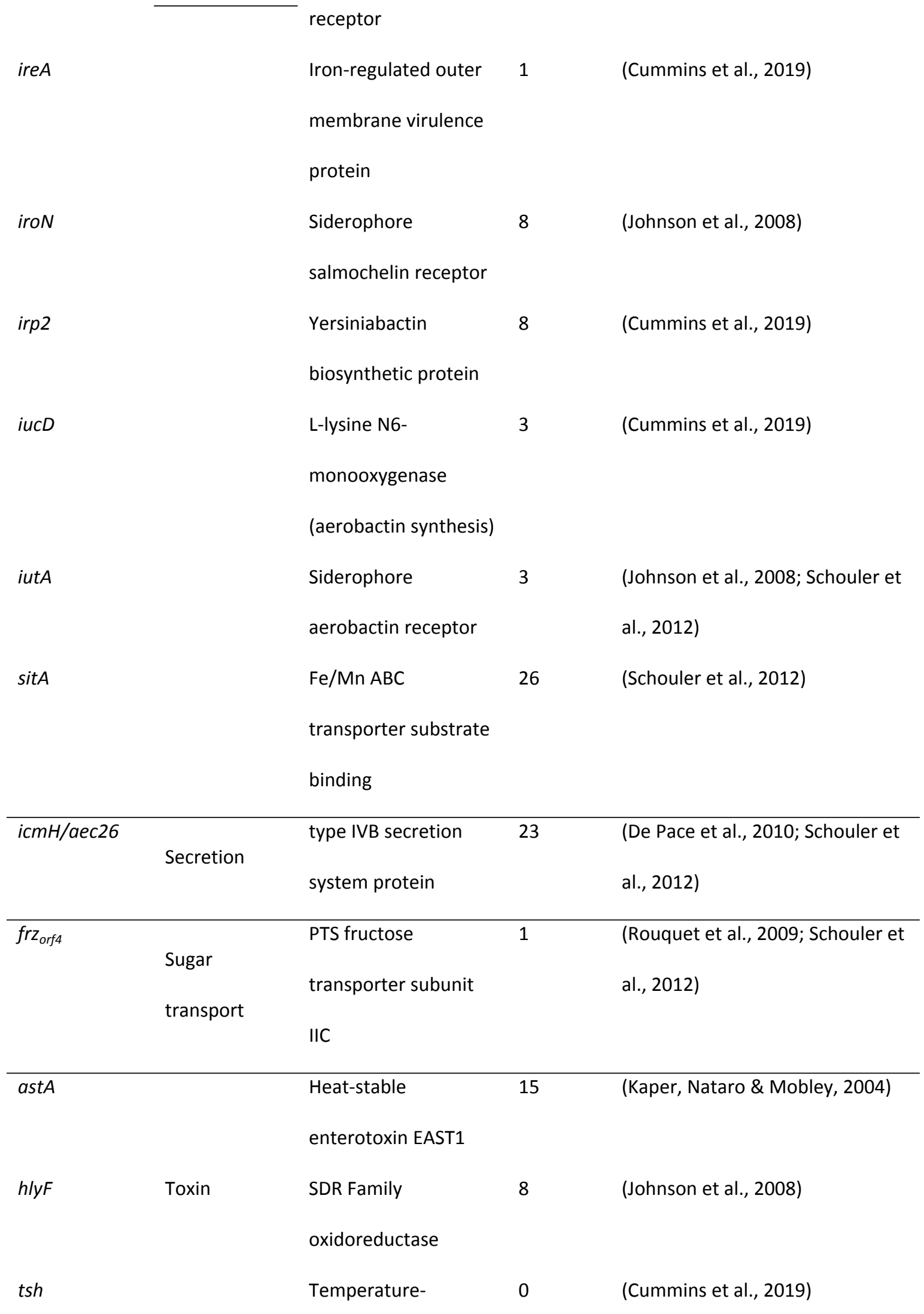




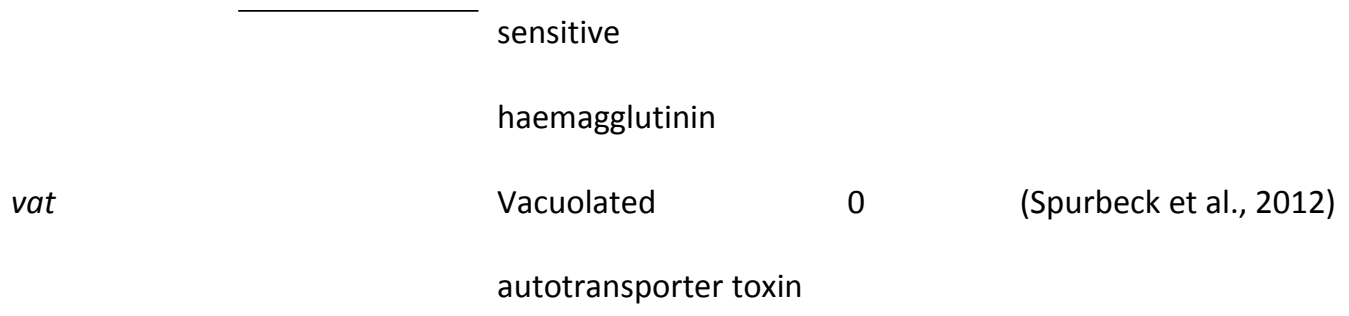

4 
Figure 1

Summary of the major stages of sample collection, processing and data analysis and the primary programs and databases used.

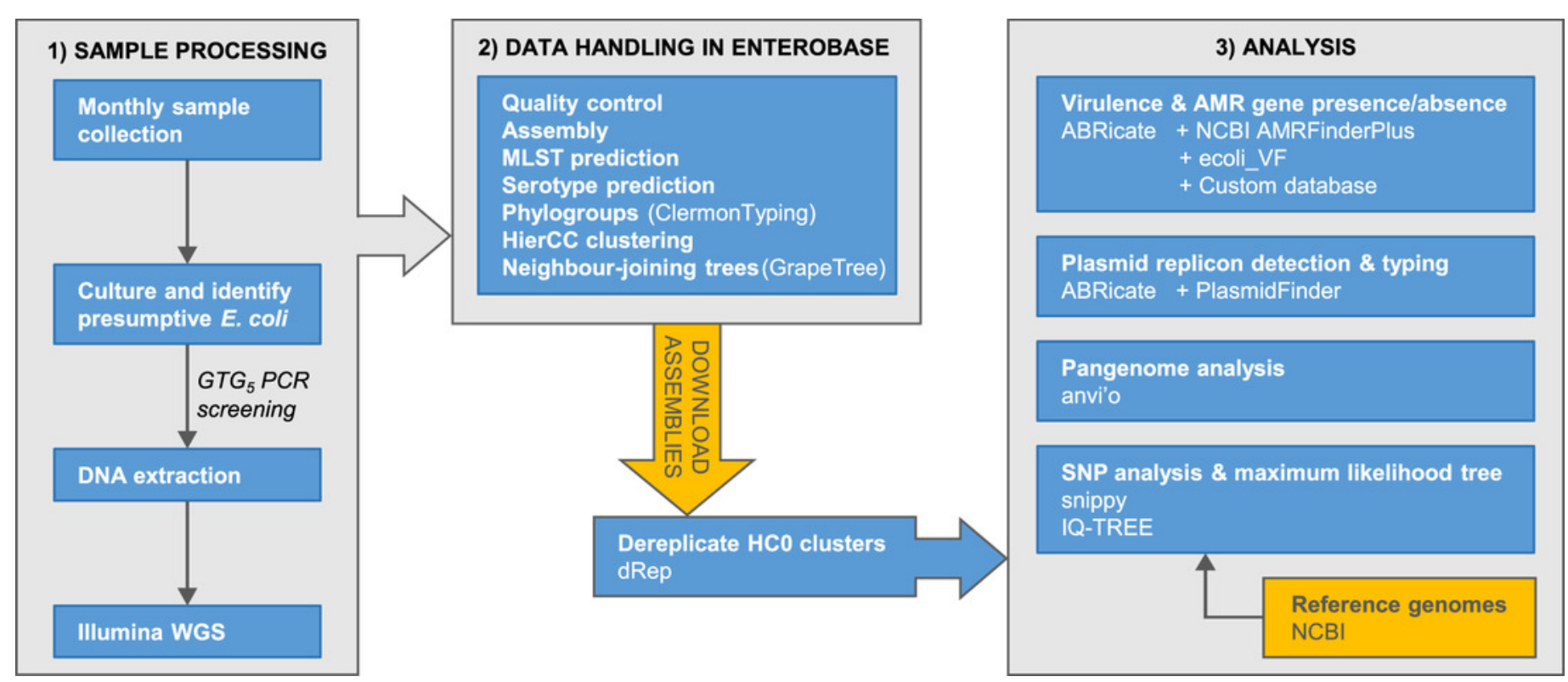




\section{Figure 2}

Core SNP maximum likelihood tree of the final 81 Escherichia isolates, including reference sequences for every Escherichia species and all phylogroups of E. coli.

Names of our isolates and their reference strains are coloured according to their species/phylogroup. Names of reference strains for clades not found in our samples are coloured black. Reference strains are named with their species and strain name, with phylogroups given in parentheses. Salmonella bongori and Salmonella. enterica ser. Typhi are included as outgroups. 


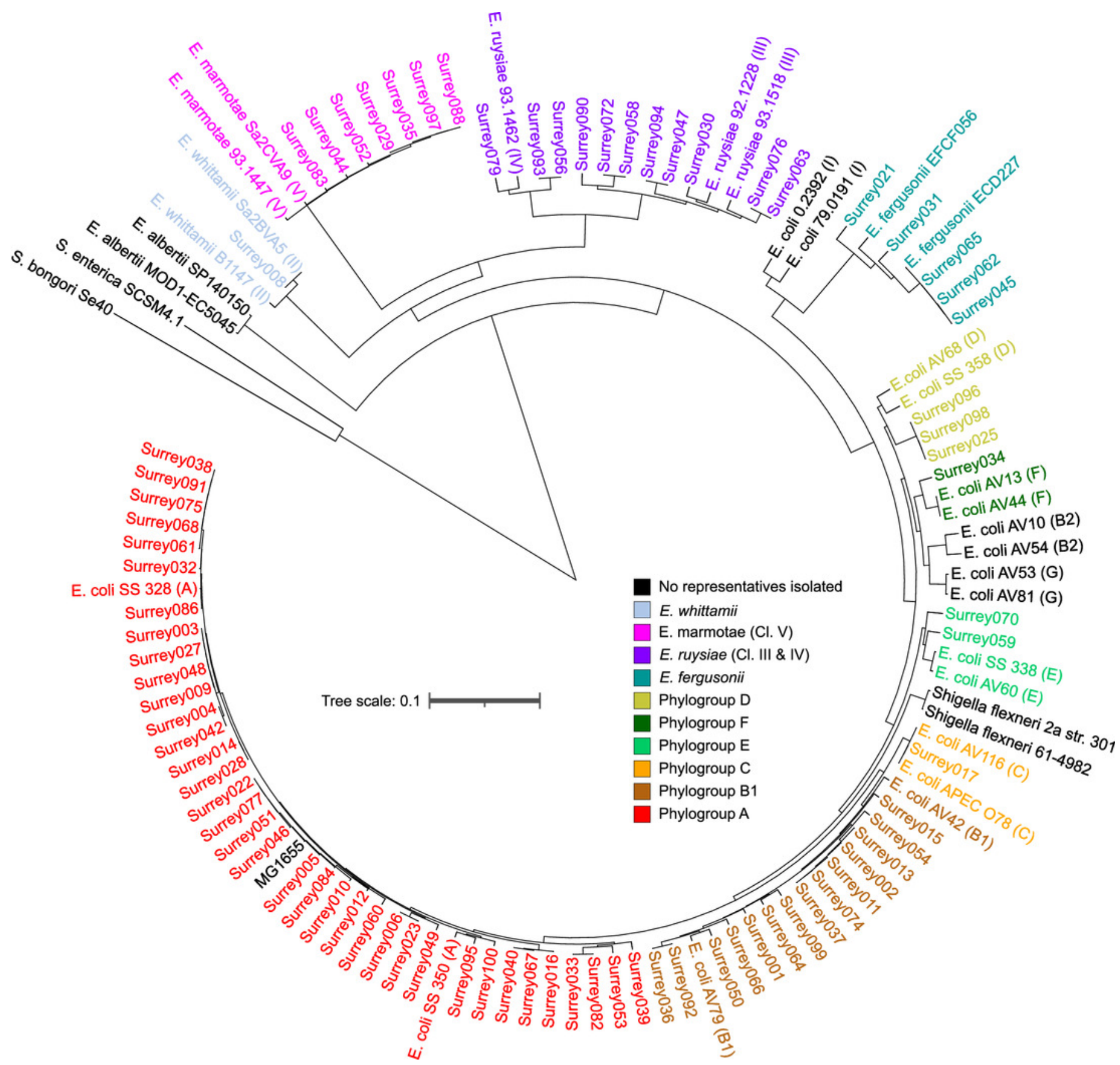




\section{Figure 3}

Anvi'o representation of the Escherichia pangenome showing 81 Escherichia isolates recovered from chicken faeces and 33 publicly available reference Escherichia isolates from poultry species.

Each layer represents a single genome, with black colouring signifying the presence of a gene cluster. Gene clusters are organised according to their distribution across the genome, with co-occurring genes shown closer together. The heatmap at the top-right of the image represents average nucleotide identity across all included genomes, with darker red colours indicating a higher percentage of average nucleotide identity. Assigned phylogroup is shown by the colour bar on the right of the image. Singleton gene clusters (present only in one genome; $n=3,641$ ) are highlighted in blue while core gene clusters (present in all genomes; $n=2,449$ ) are shown in green. 
C $(3)$

B1 (16)

- E. fergusonii (7)

E. marmotae (9)

E. ruysiae (CC4) (4)

E. ruysiae (CC3) (10)

E. albertii (2)

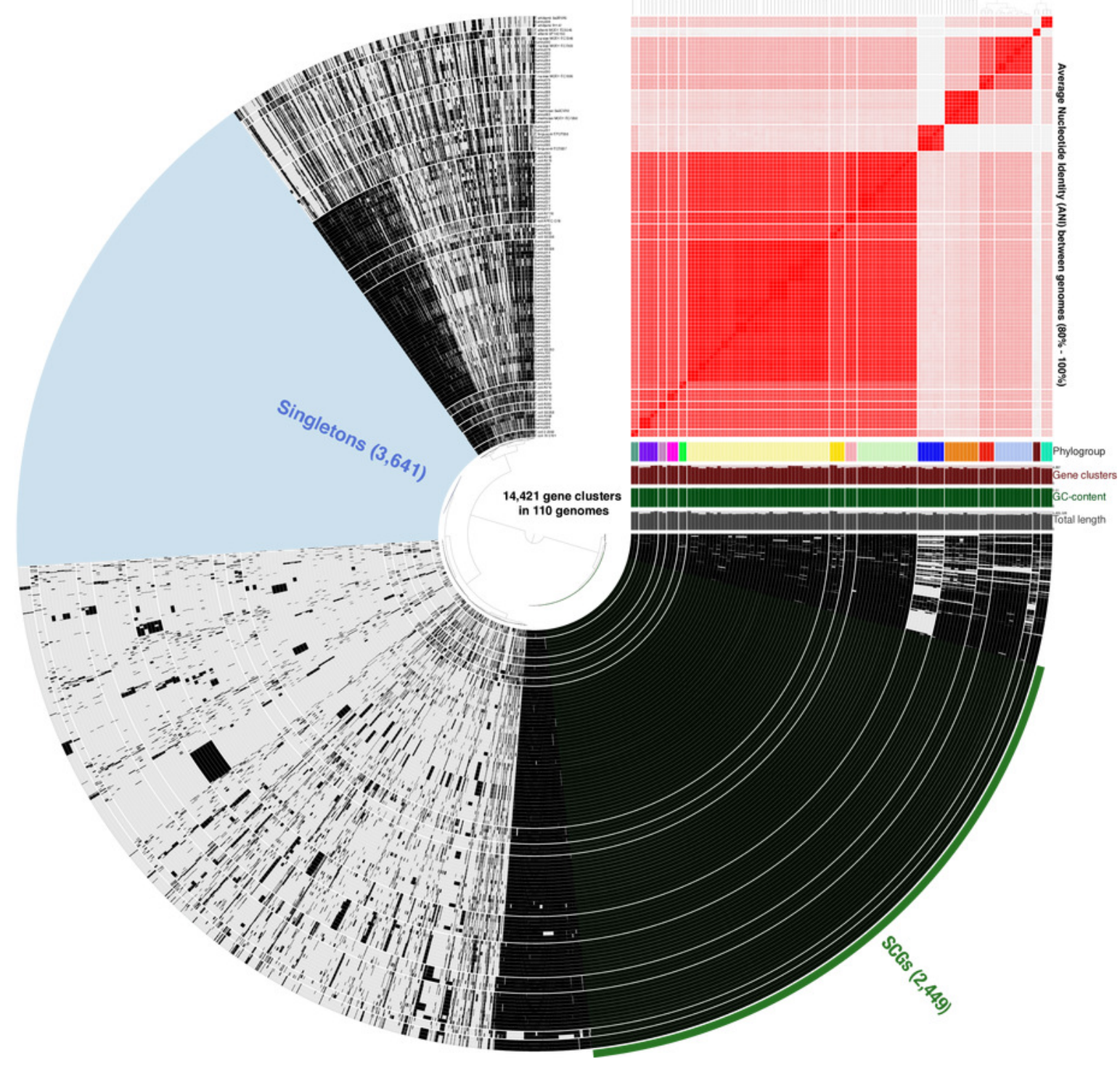


Figure 4

Summary of the plasmid replicon contents of 81 Escherichia isolates.

(A) Number of isolates containing 0 - 7 distinct plasmid replicons from the PlasmidFinder database. (B) Frequency of each plasmid replicon identified from the PlasmidFinder database. Some isolates contained $>1$ of the same replicon type (see main text). 


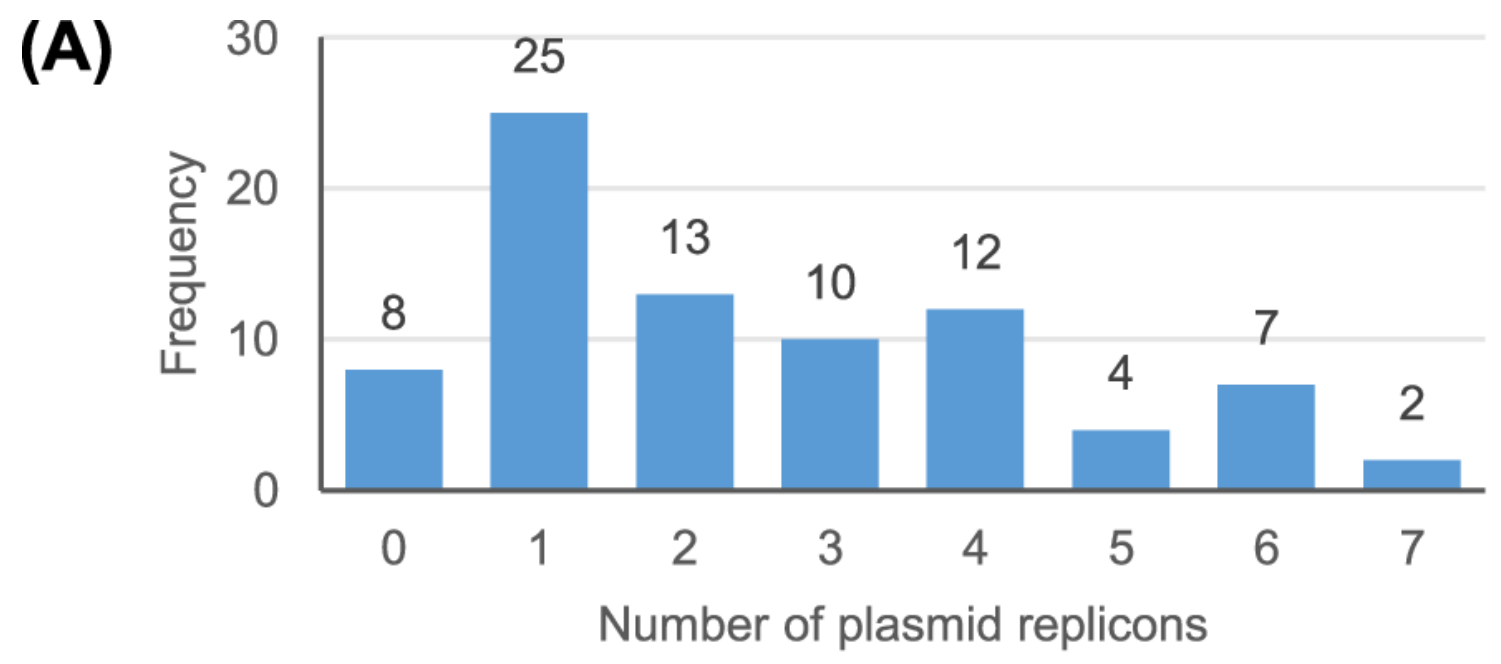

(B)

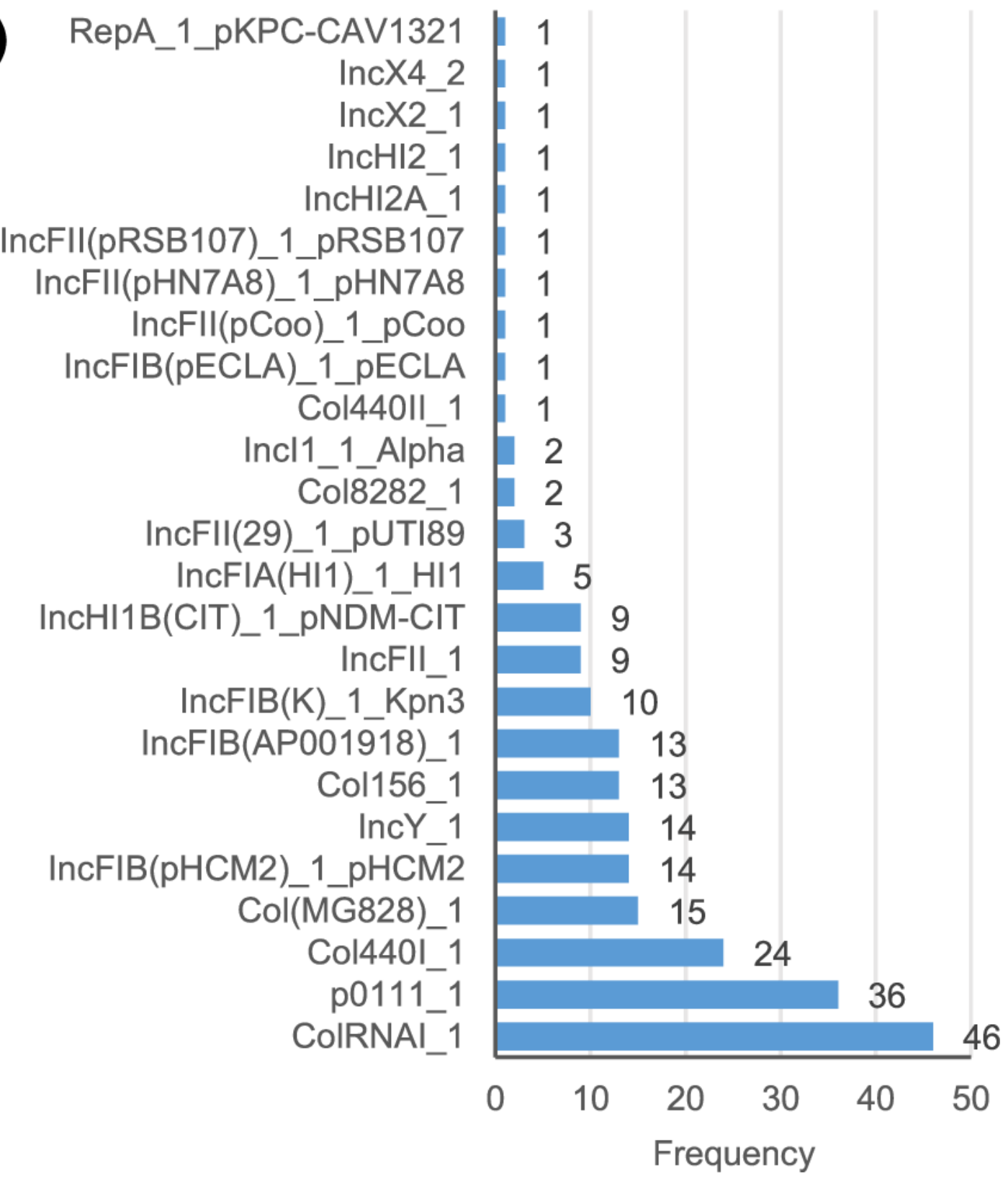




\section{Figure 5}

Presence (solid squares) and absence (open squares) of predicted antibiotic resistance genes, virulence-associated genes and plasmid replicons in the final 81 isolates.

Isolates are arranged according to the phylogenetic tree shown in Fig. 2 with their names coloured according to phylogenetic group. Ew, E. whittamii; Em, E. marmotae; Er, E. ruysiae; Ef, E. fergusonii; $A-F$, E. coli phylogroups. Virulence-associated genes shown here are from the custom panel of APEC-associated genes. Members of the panel not represented here were not present in any isolates. Plasmid replicon and predicted antibiotic resistance gene data have been condensed to show major classes. Therefore, presence indicates $\geq 1$ members of that class were detected. Full gene and plasmid detection data are available in Tables S5 - S7. 


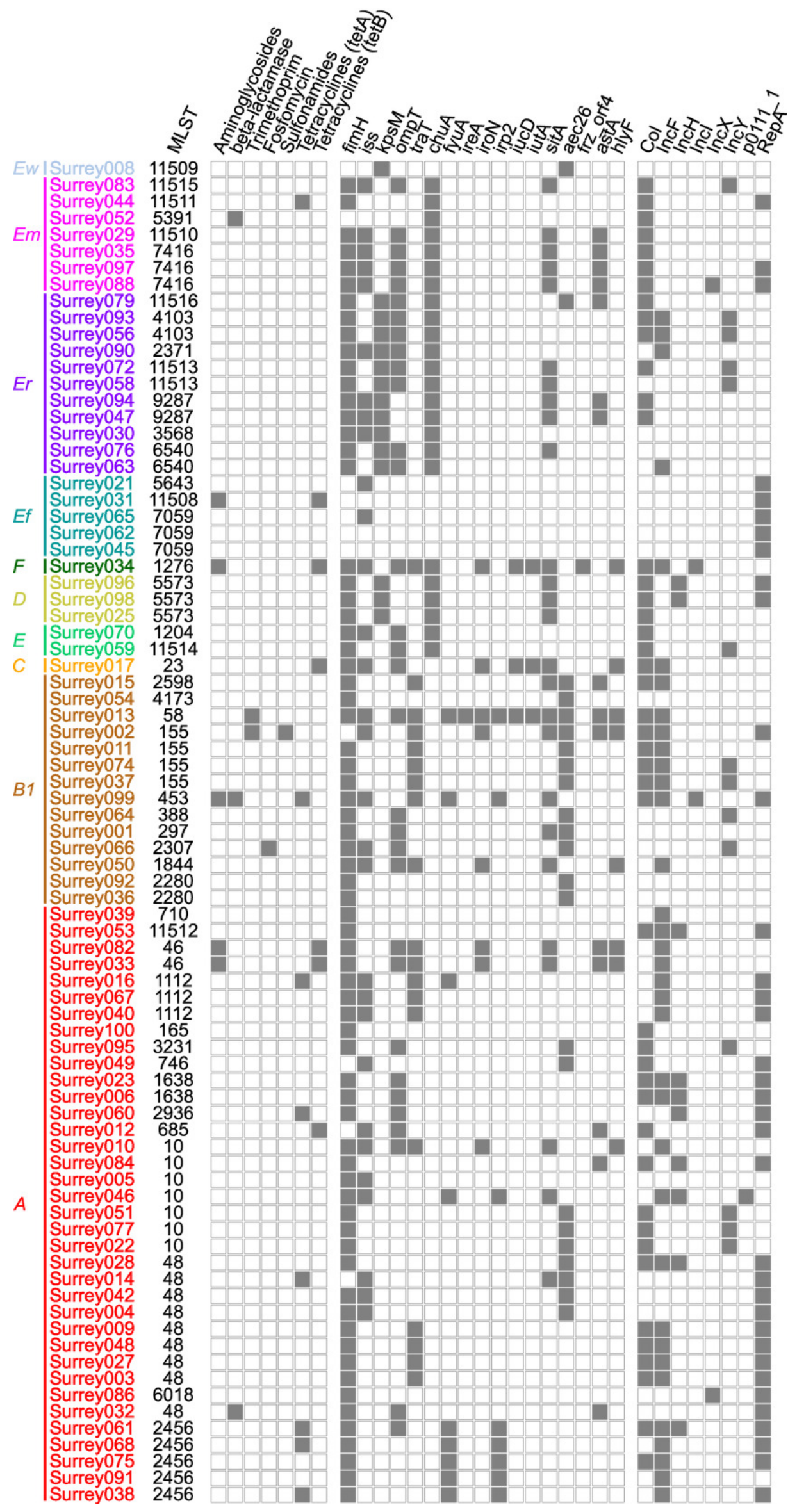

Peer) reviewing PDF | (2021:11:67677:1:0:NEW 30 Dec 2021) 